\title{
La mise en récit de l'hégémonie occidentale dans Soie et fer, du Mont-Liban au canal de Suez de Fawwaz Traboulsi ${ }^{*}$
}

\author{
Inès El Sérafi
}

\section{Résumé:}

Dans son roman Soie et fer, du Canal de Suez au Mont-Liban, paru en arabe en 2012, puis en français en 2017, l'historien libanais Fawwaz Traboulsi dénonce les agissements des deux grandes puissances occidentales (française et anglaise) au Levant au $19^{\text {ème }}$ siècle. En braquant la lumière sur la mise en œuvre de ce qui sera considéré comme « la plus grande dépossession dans l'Histoire de l'Égypte » (Raf ie al Tahtawy), ou le projet du Canal de Suez, d'une part et l'attisement d'une guerre civile qui se prolongera sur plusieurs décennies autour du Mont-Liban, d'autre part, Traboulsi tente tout à la fois de démonter et de dénoncer les rouages de la gigantesque machination impérialiste. À travers une alternance de récits d'événements et de biographies, en établissant un parallélisme entre les deux espaces de la domination, l'Égypte et le Mont-Liban, et les deux forces rivales, la France et l'Angleterre, l'auteur parvient à suggérer des associations entre des événements qui semblent sans rapport à première vue et à produire un sens, celui de la progression d'une hégémonie.

Mots-clés : Histoire connectée, saint-simoniens, Enfantin, chroniques, narration alternée, portraits-charges, Levant, Canal de Suez, Méditerranée, hégémonie.

$$
\text { ملخص باللغة العربية }
$$

يعرض فواز طرابلسي في كتاب ناريخي ليس كمثيله أسماه "حريز وحديا من جبل لبنان الى قناة السويس" حوليات ودوريات لشهادات وحكايات تسرد تاربخ الهيمنة الغربية على الشرق في القرن التاسع عشر • شخوص الكتاب الحقيقية تأخذنا من منطقة هيمنة الى الأخرى عبر صناعة الحرير في جبل لبنان تلك المنطقة المحتقنة التي حافظت علي جزوة

(*) Bulletin of the Faculty of Arts Volume 80 Issue 4 April 2020 
الصراع فيها القوة الغربية وخصوصا إنجلترا حتى تحكم سبطرتها عليها، الى مصر التي اختارها السان سيمونيان لتكون منطقة نفوذ فرنسية. يستعرض الكاتب المؤرخ عبر مكاتبات

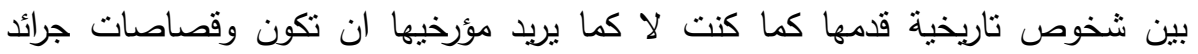

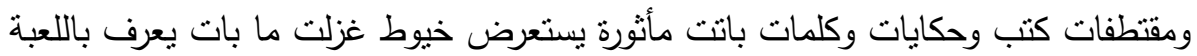
العظمى في الثرق.

Soie et fer, du Mont-Liban au canal de Suez, publié en arabe en 2012, puis traduit en français en 2017, est un récit de l'historien libanais Fawwaz Traboulsi. Écrivain et traducteur, Traboulsi enseigne l'Histoire et les Sciences Humaines à l'Université américaine de Beyrouth. Professeur invité dans nombre d'universités aux États-Unis, en Égypte et en Autriche, il a surtout pour champ d'intérêt l'histoire arabe, la pensée politique, la culture populaire et les mouvements sociaux. Auteur de plusieurs ouvrages, dont une histoire du Liban contemporain (A History of modern Lebanon, 2007), il a également traduit des œuvres de Karl Marx, d'Antonio Gramsci, d'Issac Deutscher et d'Edward Said. Rédacteur en chef depuis 2012 d'une revue culturelle trimestrielle - Bidâyât (Débuts) - il pratique par àcoups l'écriture littéraire.

Pour Soie et fer, Traboulsi refuse l'appellation de «roman historique », lui préférant celle de «récit historique ». Une ambivalence s'impose depuis le seuil, celle d'un texte qui offre une lecture double: en tant que récit historique, d'où la riche matière documentaire utilisée pour la rédaction (une liste bibliographique bien fournie, à la fin du livre, l'atteste) et en tant qu'œuvre où l'auteur recourt aux effets littéraires et romanesques (telles la descriptioncharge, l'ellipse, l'ironie, la juxtaposition, l'accumulation, la mise en parallèle...) pour raconter des événements avérés. En mettant l'accent sur la véracité des faits racontés, autrement dit sur l'ancrage historique du texte, Traboulsi prend le parti de les traiter librement. Pour décrire son récit, l'auteur parle d'un texte en forme de «chroniques », inspirées de toutes sortes d'écrits historiques, documentaires et 
littéraires : archives, correspondances, témoignages, mémoires, récits de voyage, etc., qui se manifestent sous forme de fragments juxtaposés. Si la fiction n'habite pas les faits relatés, un effet de romanesque se dégage de leur évocation. Les traductrices de Traboulsi parlent d'une familiarité qui a fini par s'établir entre l'auteur et les personnages de son récit au fil de la lecture ${ }^{2}$. Dans Soie et Fer, ces derniers ressemblent peu à ceux qui peuplent d'autres types de textes appartenant à la sphère historiographique stricto sensu. L'angle adopté par Traboulsi est celui d'une certaine forme d'écriture nouvelle de l'histoire, qui n'est pas sans rappeler l'Histoire connectée ${ }^{3}$.

En tant qu'approche historiographique, l' « histoire connectée » est née, on le sait, d'une critique des cadres d'analyse fixes et d'un refus du déterminisme historiographique eurocentriste. Ce qui la caractérise essentiellement, c'est la multiplicité d'angles d'approche et de points de vue, l'établissement de rapports et d'associations entre les événements et le fait d'admettre une périodisation autre que celle de l'historiographie construite depuis des décennies, notamment par les historiens occidentaux. Soie et fer illustre cette approche et propose une vision autre des relations Orient-Occident au $\mathrm{XIX}^{\mathrm{e}}$ siècle en ramenant les divers événements, qui peuvent sembler épars à première vue, à un même projet hégémonique. En diversifiant les archives, en les croisant, en acceptant de nouvelles interprétations, Traboulsi retrace la trame d'une course expansionniste qui oppose les deux empires, français et anglais, entre le Liban et l'Égypte, pendant cette période charnière dans l'histoire de la domination occidentale du Levant que fut le $\mathrm{XIX}^{\mathrm{e}}$ siècle.

Neuf chapitres, dont le premier s'ouvre en 1806 « Sur la côte espagnole », et des «Épilogues ${ }^{4} »$ composent la totalité du récit à la manière d'un grand puzzle dont les pièces s'assemblent au fil de la lecture. Depuis le seuil de l'œuvre, des fils de «soie » et de «fer» lient le Mont-Liban au canal. Partant d'un espace situé sur la côte 
orientale de la Méditerranée, ils visent un projet dont le nom s'affiche dans l'intitulé, le canal de Suez, et un espace présent en filigrane dans la trame du récit, l'Égypte. La « soie » et le «fer» de Traboulsi peuvent se prêter à diverses interprétations où le sous-entendu a sa place. Dans l'impossibilité de reproduire la paronymie suggestive propre à l'intitulé arabe «Harîr wa Hadîd »" le titre choisi par les traductrices invite à penser à une relation qui ne semble pas évidente d'emblée pour un lecteur francophone. De la « soie », il sera question dans le texte qui fait de l'industrie soyeuse l'emblème de la condition ouvrière et féminine en Europe, mais aussi au Levant, à l'aube des doctrines communistes. Quant au (chemin de) «fer», il constitue une allusion aux projets de communication entre Orient et Occident qui ne vont pas tarder à foisonner, projets de liaison qui s'avèrent des projets de contrôle. Dans le chapitre éponyme du roman « De fer et de soie », l'alliance entre les deux matériaux est établie explicitement pour la première fois dans la citation d'une formule attribuée à l'émir Abdelkader lors de la signature de la trêve avec la France : «[... l'Émir dira : "L'homme est fait de soie et de fer", faisant ainsi allusion à la nécessité d'alterner guerre et diplomatie. Cette phrase, parvenue à la presse française, la séduit ${ }^{6} »$.

Or cette phrase «séduisante » résume, on le verra, un vaste programme de domination où à l'usage de la guerre et de l'affrontement se conjugue une stratégie placée sous le signe de l'art de l'esquive et de la dérobade : «soie » et «fer » renvoient donc également aux procédés de la mainmise occidentale.

Dans sa deuxième partie, l'intitulé polysémique de l'œuvre met l'accent sur une dualité spatiale : Mont-Liban d'une part, Égypte de l'autre. Il suggère également en filigrane que le canal de Suez serait l'aboutissement, voire la fin, de ce qui se prépare au Mont-Liban. C'est pourquoi, dans les pages qui suivent, nous partirons de la construction de l'espace, pour examiner comment Traboulsi organise 
sa reconstitution / dénonciation du projet hégémonique européen en Orient, en misant à la fois sur un système particulièrement original des personnages et sur une trame narrative qui traduit, sur le plan du récit, les entrelacs de la trame complexe qui s'ourdit dans les hautes sphères de la politique européenne à l'encontre de la région.

\section{Un même projet et des espaces divers}

Dans le récit, le cadre spatial de Soie et fer se révèle étonnamment pluriel. Depuis le titre sont inscrits les champs d'action des deux forces impérialistes. Le projet comprend le Cham (terme arabe désignant la grande Syrie ou la région syro-libanaise et la Palestine) et l'Égypte, mais aussi ce qui se trouve sur le chemin qui les relie. Si le récit s'ouvre en Europe, sur une côte espagnole où Français et Anglais rivalisent pour la maîtrise de l'espace méditerranéen, il se clôt en Orient, par la «Confluence des deux Mers » (titre du chapitre 9) en Égypte, où l'inauguration du canal de Suez en 1869 vient couronner l'aboutissement du projet hégémonique. Un espace de la machination, décentré et éclaté, couvre les deux rives septentrionale et méridionale de la Méditerranée. Si l'Orient est le théâtre des grandes manœuvres occidentales, l'Europe et Constantinople sont le lieu où elles se combinent. Karl Marx, («le docteur allemand» de Soie et fer), alter ego légèrement bouffon de l'auteur, dénonce le complot et ne voit d'ailleurs partout que machinations :

Le Docteur suit avec une extrême attention l'évolution des relations entre l'Europe et le sultanat ottoman. Sa position révèle l'ampleur de son hostilité au descendant de Napoléon. Il accuse la Russie et la France de semer la discorde en Orient sur les plans politiques et religieux. Il accuse l'empereur des Français et le tsar de Russie de rechercher tous deux une guerre extérieure afin de se dérober à leurs problèmes internes. Selon lui, tous deux intriguent afin de se partager l'Europe. Quant à Lord Palmerston, il l'accuse à son tour d'avoir armé les druzes du Mont-Liban en 1846, et, la même année, d'avoir 
contracté un accord avec le tsar ayant conduit en pratique à « annuler la domination ottomane sur les tribus libanaises insoumises, leur offrant une indépendance de façade qui, avec le temps et grâce aux manœuvres des intrigants étrangers, ne leur a fait récolter que du sang $\gg^{7}$.

La France, au sein d'un conflit avec les grandes puissances européennes, et l'Angleterre, puissance rivale, sont les acteurs de la mainmise. Plus tard, au tournant du siècle, lorsque tout ce qui se passe au Levant se mue en affaire proprement occidentale, c'est l'Europe tout entière qui devient agent de la domination. Le chapitre 5, «Orientalisation-Occidentalisation », est un chapitre bien européen. Traboulsi y dépeint le «Printemps des peuples». En 1848, un mouvement révolutionnaire et populaire agite tout le continent, quand « un vent de liberté souffle sur les villes et les campagnes d'Europe ${ }^{8}$ ». Le décentrement de l'espace, résultat d'un élargissement du champ de vision de l'auteur, confère aux événements un contexte qui dépasse le lieu où ils se déroulent. L'échec des soulèvements libéraux en Europe explique, voire provoque, le rythme accéléré des actions expansionnistes au Levant ${ }^{9}$. Le coup d'État du 2 décembre 1851, qui instaure le régime autocratique de Napoléon III en France, prépare le terrain pour le projet du canal en Égypte. Aussi, le texte progresse-t-il essentiellement par un aller-retour entre les deux rives de la Méditerranée. Entre ces espaces de la domination un parallélisme finit par s'imposer, que renforce le respect d'une chronologie diachronique. La temporalité du texte, régie par une périodisation méditerranéenne, offre une nouvelle vision des pays dominants et dominés, et c'est là l'une des originalités de l'œuvre que de produire, à travers le croisement d'espaces et de cadres temporels, un contre-récit. Soie et fer s'ouvre en 1806 sur une Europe mobilisée pour contrer l'appétit expansionniste de Bonaparte. En Égypte, Muhammad Ali (surnommé le nouveau Napoléon, «l'homme destiné à parfaire la mission du grand empereur », p.72) est au pouvoir depuis 1805. Il est l'homme 
fort de l'Orient et suscite, à son tour, la défiance des puissances européennes qui agissent sans plus tarder. Au Mont-Liban, les signes d'une tension que les Anglais maintiennent afin de contrôler le Levant ne tardent pas à se manifester.

La narration alternée est soutenue par une galerie de portraits qui orchestrent la mise en récit du projet hégémonique. Vue de part et d'autre, l'Histoire autre que Traboulsi expose est une suite d'actions attribuées à des hommes. L'approche démythificatrice de ces « hommes-là » permet de ramener l'histoire à une dimension humaine tout en donnant à voir les dessous des événements. Sur un autre plan, c'est par la peinture des personnages que le récit historique de Traboulsi flirte avec le romanesque. La technique de l'auteur est en somme ambivalente : exacerber les traits avérés de ses personnages en retenant essentiellement ce qui les apparente à des héros de fiction pour rester au plus près d'une « histoire connectée » qui permette au lecteur d'accéder à une autre version des faits. La démythification est le mot d'ordre de ces contre-portraits.

\section{Biographies biaisées et contre-portraits}

Les personnages de premier rang de Soie et Fer sont doublement excentriques: fantasques, ils sont situés en marge de la sphère historiographique du $\mathrm{XIX}^{\mathrm{e}}$ siècle. Autrement dit, ils ne sont pas toujours ceux que 1'Histoire eurocentriste dépeint en tant qu'acteurs principaux de la scène politique. En traçant leurs portraits, Traboulsi est amené du même coup à les placer au cœur du grand projet du siècle. Son parti pris étant de traiter librement les faits, ces portraits, qui virent souvent au portrait charge ou contre-portrait, sont des biographies plus ou moins biaisées, c'est-à-dire des « récits où la question de la vérité est suspendue par d'autres enjeux : la nécessité de mémoire, l'exemplarité, le jeu romanesque, l'éloquence, l'émotion collective, la piété familiale ou religieuse, etc. ${ }^{10}{ } \gg$

Le but de Traboulsi est de cerner les personnages-outils de la 
domination, à travers une écriture outrée et dénonciatrice. Grossis sous l'effet d'une peinture au vitriol, ces personnages caricaturés mettent à nu la machination occidentale. Ce qui s'esquisse au travers de l'exagération rhétorique (avec sa panoplie de figures : hyperbole, litote, accumulation...), c'est paradoxalement la vérité des événements et des agissements exercés par l'impérialisme occidental dont ces personnages sont les instruments. Se livrer à une déformation stylistique de personnages historiques tels que lady Esther Stanhope, la célèbre aventurière anglaise, Enfantin, Lamartine ou Lesseps, c'est aussi prendre sa revanche, par le rire et l'excès, sur un acte d'asservissement et élaborer, a posteriori, une culture de la résistance $^{11}$. Les portraits, qui ne versent ni dans le symbolisme (Ferdinand de Lesseps, par exemple, n'est pas à lui seul la France coloniale), ni dans l'allégorisme (il n'est pas non plus la convoitise impérialiste personnifiée), sont régis par la juxtaposition de traits sans commentaires explicatifs. Or chez Traboulsi, la juxtaposition abrupte est la figure qui rend le mieux son geste de chroniqueur et d'historien à la recherche de liens entre les événements. Par l'accumulation, il vise une autre fin : accentuer l'effet d'incongruité sous l'enseigne de laquelle il place ses personnages occidentaux.

Le portrait de Prosper Enfantin est le plus représentatif de cette écriture ironique. Enfantin appartient à la catégorie des « conquérants » français $\mathrm{du} \mathrm{XIX}^{\mathrm{e}}$ siècle, à savoir les tenants d'un discours universaliste et «fraternel » qui, s'armant de grandes idées utopiques, agissent en réalité dans le cadre de la rivalité francobritannique pour la maîtrise du Levant. Férus de grandiloquence, ils s'expriment avec force stéréotypes, empreints d'une idéologie « orientaliste» (selon l'acception d'E. Said) dont le propre est de hiérarchiser les rapports entre Orient et Occident :

Les saint-simoniens placent la notion de « civilisation » au cœur de leur doctrine. Or, pour que le monde entier en profite, il faut le 
quadriller de chemins de fer, de centrales électriques, de mine d'or et d'argent. Selon eux la Méditerranée est le principal bassin de civilisation, «le lit nuptial de l'Orient et de l'Occident» pour reprendre les mots de Michel Chevalier, auteur de la théorie du «système de la Méditerranée ». Le couple Orient-Occident est l'équivalent de l'union, dans la symbolique chrétienne, de l'esprit et du corps. L'Occident est l'esprit ; l'Orient, le corps. Or l'Égypte est le portail de l'Orient $^{12}$.

Prosper Enfantin est un personnage central de Soie et fer. Pour Traboulsi, c'est bien lui l'homme du canal, plutôt que Lesseps. En disciple fidèle de Saint-Simon, lui-même caricaturé et montré dans le récit sous la figure d'un obsédé, Enfantin prend sur lui de diffuser ses théories industrielles et socialistes, mais aussi celles qui concernent la communication des mondes à travers force ponts, barrages, canaux et chemins de fer - et de les mettre en œuvre. Un portrait satirique d'Enfantin en fait le personnage le plus extravagant de l'œuvre :

La place est désormais libre pour la mégalomanie d'Enfantin qui se prend pour l'élu de Dieu, le semblable du Christ. Il est porté aux nues par ses adeptes qui le surnomment tour à tour «Loi vivante », «Élixir d'amour», « Cathédrale d'altruisme », « Maître du monde ». « Notre maître Jésus vit en lui », disent-ils. « Le saint-simonisme était une simple doctrine, il est désormais une religion. » Ils se lancent à la recherche de son autre moitié, la mère ${ }^{13}$.

L'antithèse est au nombre des figures dont use Traboulsi pour le portrait outrancier $\mathrm{du}$ «Petit père français du monde». Le récit mentionne une de ses lettres où, ne cessant de divaguer, il est montré sous un jour plutôt insolite : «Je ne pense pas, dit-il, que je sortirai d'ici vêtu d'une robe, mais je ne serais pas surpris que le monde me croie métamorphosé en femme... ${ }^{14} \gg$. Comble de l'excentricité, à laquelle Traboulsi le réduit : dans le récit, Enfantin a pour principale activité mentale le rêve. Au sens propre du mot : 
Enfantin rêve à nouveau de percer. Cette fois-ci, il s'agit de lignes de chemin de fer. Dans son sommeil, il voit les rails traverser la France du nord au sud en passant par Paris, Lyon et Marseille. Il entreprend alors de fonder l'Union du chemin de fer de Paris à Lyon, avec un capital de départ estimé à 70 millions de francs ${ }^{15}$.

Traboulsi intègre le projet du canal au nombre des rêves du Maître, de ceux que les saint-simoniens se transmettent au-delà même de la survie de leur mouvement. Les projets ambitieux, telle «la fameuse PLM, la Compagnie des chemins de fer Paris-LyonMéditerranée », et les autres dont d'anciens amis se chargent - cas du canal de Suez par Lesseps -, auraient d'abord été échafaudés pendant son sommeil. Après les avoir vus en songe, le « Père » n'a plus qu'à travailler, sans relâche. Outre le caractère fantaisiste du personnage ainsi grossi, Traboulsi fait du canal, hantant les esprits, un projet ancestral de domination. Telle est la symbolique du rêve dans Soie et fer. Ces « rêves » tour à tour «millénaires » ou « collectifs », sont des projets qui attendent d'être réalisés. Aussi, le creusement d'un canal unissant les deux mers traduit-il une obsession française, remontant au moins à Bonaparte, d'une domination sur la Méditerranée qui passe par l'Égypte, «portail de l'Orient ». Ce sera la destination du premier voyage saint-simonien outre-mer.

\section{Les entrelacs de l'intrigue}

Le récit nous montre Enfantin embarquant pour l'Égypte en 1833, à la recherche du Christ-femme, de la «Mère » avec laquelle il est censé formé un couple mystique. Il est à la fois accompagné et précédé par une escouade de saint-simoniens, les «Compagnons de la femme ». Or, un extrait de presse que cite Traboulsi annonce que le voyage répond à un autre motif :

L'Écho de la fabrique, un journal proche des saint-simoniens, salue leur passage à Lyon avant leur départ. Dans le numéro du 15 septembre 1833, on peut lire que leur expédition a pour ambition de 
« faire un chemin ou un canal à travers un désert de plus de 30 lieues afin de réunir la mer Rouge à la Méditerranée et de rendre ainsi l'Europe toute voisine de l'Inde ; voilà le travail qu'avec les hommes d'une grande valeur, il est vrai mais sans argent, le père Enfantin veut entreprendre $\gg{ }^{16}$.

Les architectes des grands projets civilisationnels sont désargentés. En Égypte, les saint-simoniens sont à la recherche de financement. Leur mission s'inscrit d'emblée dans le prolongement de l'expédition napoléonienne de 1798. Au Caire, Enfantin jette son dévolu sur le vice-roi, et sollicite sa rencontre. Muhammad Ali, réticent, refuse le projet : il craint pour l'Égypte le sort de la Sublime Porte, fragilisée par le Bosphore. À défaut d'un canal, et d'un chemin de fer, Enfantin obtient le droit de construire un barrage (jamais achevé), à Damiette. La mission saint-simonienne se place sous le signe du «premier conquérant de l'Égypte ${ }^{17}$ », alias Bonaparte. Ferdinand de Lesseps, c'est sa première apparition dans le texte, fait régulièrement partie des festivités saint-simoniennes régulées par un calendrier napoléonien. Bientôt un autre rêve « du maître » livre le secret du canal :

Au réveil, Enfantin sait à l'évidence que la faille universelle se situe dans la région de Suez. C'est là-bas que doit se réaliser le rêve millénaire reliant la Méditerranée à la mer Rouge : «Suez est le centre de notre vie de travail. Là, nous ferons l'acte que le monde attend pour confesser que nous sommes mâles! C'est à Suez que les eaux de l'Orient féminin se mêleront à celles de l'Occident masculin. ${ }^{18}$

Le projet prend forme notamment avec la désignation du lieu : le canal sera de Suez. En le situant, Enfantin lui aurait donné du même coup son nom. Mais l'aventure orientale des saint-simoniens tourne court. S'ils quittent l'Égypte «sans avoir rien ouvert ni bouché de concret », comme dit plaisamment Traboulsi ${ }^{19}$, Enfantin n'oublie pas le projet du canal. De retour en France, il troque à jamais son 
messianisme de façade contre un impérialisme cru et élabore toutes sortes de théories sur l'émancipation d'une Égypte qui s'était interposée entre ses rêves et lui :

Devant l'écroulement de ses rêves, le Petit père français du monde se met à raisonner de façon diamétralement opposée. Désormais, il pense que l'émancipation de l'Égypte doit passer par l'éradication de l'espèce turque, au moyen d'une occupation militaire franco-britannique du pays ${ }^{20}$.

Traboulsi recourt à l'ellipse et aux techniques de raccourci pour mettre à nu la duplicité du personnage. Au nombre des procédés dénonciateurs qui dévoilent la vérité colonialiste des saint-simoniens, le passage sans transition des rêves du « Maître » à ses théories accuse le trait. L'universalisme, la fraternité et l'union entre les peuples ne sont plus qu'un masque derrière lequel se dissimule le cynisme de l'homme qui se met à prêcher haut en faveur du colonialisme. Les grands discours et la spiritualité d'Enfantin font place à un pragmatisme cynique.

En faisant l'économie de toute explication, en présentant le changement comme une conséquence normale de l'échec essuyé en Égypte, Traboulsi passe d'un état à l'autre de façon accélérée, ce qui accentue l'antinomie abrupte qui fonde le personnage. Sitôt dit, sitôt fait, donc. La France et l'Angleterre œuvrent au dépècement de l'empire ottoman, c'est-à-dire du Levant et de la Turquie. En Algérie, Enfantin improvise un nouveau modèle de colonisation, à la fois « moderne » et « pacifique », à travers « l'occidentalisation ${ }^{21}$ », le tout sur fond d'étude ethnographique. Sur l'autre rive de la Méditerranée, les théories dues aux «démocraties colonisatrices » pleuvent: Alexis de Tocqueville, Lamartine et Victor Hugo s'en font les chantres. Traboulsi met sous les yeux de son lecteur des bribes de leurs discours. Ses commentaires sont minimalistes. Au service de la dénonciation, la juxtaposition comme figure permet à l'auteur de 
cibler la dualité propre à un esprit occidental intéressé. Il pointe comme répugnant le pragmatisme des penseurs censés emblématiser le vent de liberté et d'idées républicaines qui souffle sur l'Europe. La démocratie des grands romantiques français est une chimère. Assumant pleinement leur machiavélisme, ils le déclament dans des discours publics et des écrits sur la mission civilisationnelle de l'Occident en Orient.

Entre temps, la tension monte au Mont-Liban et les puissances européennes entrent en lice. Le chapitre IV est celui qui relate ces événements. «De Fer et de Soie », un des plus longs du récit, raconte une décennie méditerranéenne charnière : de 1837 à 1846, où, en vrac, l'Algérie s'apprête à tomber aux mains des Français, la discorde commence à germer au Mont-Liban, la défaite égyptienne d'Ibrahim Pacha met fin à l'ambition de Muhammad Ali extra muros, la question d'Orient est conclue en faveur des puissances européennes, et le canal de Suez prend la tournure d'une « affaire commerciale ${ }^{22}$ »- tout cela au profit de la France. Dans un ultime élan de pragmatisme, Enfantin, converti en industriel, oublie à tout jamais l'ancien rêve oriental du Christ-femme. Dans la même veine satirique, Traboulsi double ce changement de cap d'un « changement de costume ${ }^{23}$ » cette fois :

Le Maître change d'uniforme et remplace son austère habit monacal de Ménilmontant par le costume bourgeois des hommes d'affaires. Un grand portrait de lui, peint à l'huile, le représente vêtu conformément à sa nouvelle position, une main posée sur une pile de trois livres : la Bible, le Coran et les Enseignements de Saint-Simon ${ }^{24}$.

Enfantin est deux fois représenté : dans le texte et dans son «grand portrait [...] peint à l'huile » qui le montre tel un personnage assumant son passé saint-simonien et son présent bourgeois et capitaliste. Ce sont les grandes entreprises de communication qui se chargent de la transition entre l'ancien Enfantin et le nouveau. En 1846 , dans sa demeure « rue de la Victoire », se tient une réunion où 
sont exposés études, plans et budget du canal : la «Société d'études du Canal de Suez » est fondée ${ }^{25}$. Toujours sous-jacent, constamment en relation avec les agissements occidentaux en Orient, le projet du canal reste d'actualité, même quand il passe au second plan. L'objet de la convoitise occidentale étant le même, la rivalité entre les puissances européennes ne tarde pas à s'attiser, notamment au MontLiban, sur fond de conflit religieux. L'émiettement de la région syrolibanaise s'avérant dans l'intérêt de toutes les puissances occidentales - même de Constantinople qui, se sentant démembrée, livre sa dernière bataille -, un accord est vite conclu qui promet d'affermir la domination occidentale en alimentant toutes les tensions. Dans les derniers chapitres de Soie et fer, la «percée » devient alors un leitmotiv: toute entente entre les puissances rivales ne peut être envisagée à moins d'y associer la mise en œuvre du projet d'un canal reliant les deux mers bordant l'Égypte. Un avant-dernier chapitre, «La transaction», qui s'ouvre sur un développement intitulé « La Percée », raconte la grosse rafle que représente le projet du canal. En mettant d'accord Français et Anglais en Orient, le canal freine leur rivalité et unit leurs efforts. Le puzzle narratif commence à s'assembler, ses pièces s'emboîtent. Lesseps, qui obtient la concession en 1854, réussit là où Enfantin avait échoué, non seulement parce que, entre-temps (comme le veut l'anecdote), le wali d'Égypte est Muhammad Saïd, dont il était l'ami, mais surtout parce que Napoléon III promet aux puissances européennes de céder à ce prix l'idée de fonder un double royaume français : arabe au Machrek et catholique au Mont-Liban ${ }^{26}$ :

Il semblerait que l'Histoire ait donné raison à Bufour al-Haboul, quand il conseillait d'utiliser la menace du royaume arabe dans le dessein de le troquer. Les mauvaises langues diront que la transaction de l'empereur n'a pas consisté à échanger le royaume arabe d'Abdelkader contre le pachalik de Damas ni contre l'émirat maronite, mais bien contre une brèche dans le désert égyptien. Mais 
quelle brèche ${ }^{27}$ !

Le projet de la mainmise, dans Soie et fer, est bien double: renforcer la domination sur le Mont-Liban permettra d' "émanciper l'Égypte ». Tout prend sens et se tient, à la fois dans l'architecture de la grande saisie de l'Orient par les puissances européennes, et dans le texte qui les met en récit. L'entrelacs de la trame hégémonique se lit au niveau du cadre spatial qui nous mène de l'Algérie au Mont-Liban et vice-versa, transitant par la France et l'Égypte. En multipliant les allers-retours entre les troubles du Mont-Liban et la nouvelle voie maritime en Égypte, en resserrant ainsi le rythme de la narration, Traboulsi fait des premiers les prémices sans lesquels «la brèche » n'aurait pu voir le jour.

Le canal de Suez est bien ce deuxième volet du diptyque de l'hégémonie européenne. Traboulsi le place au cœur d'une grande spéculation. L'ancienne idée française d'un canal en Égypte n'est réalisable que lorsqu'elle intègre un projet plus grand: celui d'une Europe omniprésente en Orient. L'union entre les deux mers ne tarde pas à concilier la France et l'Angleterre, aux dépens d'ennemis communs : la Turquie, bientôt morcelée, et l'Égypte, déjà dépossédée, sans parler du Mont-Liban à jamais divisé. L'Égypte est désormais sous l'emprise de la «Compagnie universelle du Canal maritime de Suez » sise à Paris, qui hérite de celle d'Enfantin. D'autre part, en associant le Mont-Liban au jeu des puissances européennes, Traboulsi - non pas en tierce partie comme pour le canal ou l'Algérie, mais en historien libanais - explique les origines d'un conflit durable, à la lumière d'un «Great game » en Orient. Son récit s'articule autour d'un parallélisme qu'il nous suggère entre les lieux de la machination, parallélisme qui reproduit celui des deux puissances rivales. MontLiban et Égypte d'une part, France et Angleterre de l'autre : les deux rives de la Méditerranée, que d'anciens projets rêvent de relier, finiront par se rejoindre. Dans Soie et fer, Traboulsi opte pour une 
forme d'écriture (la chronique, avec des figures telles que la juxtaposition, l'accumulation, le fragment, le raccourci) et une approche (celle de l'Histoire connectée), qui favorisent la création de liens, les associations et les rapprochements. Par les allers-retours constants entre les pays dominants et dominés ainsi qu'entre les personnages campés dans une veine satirique, se crée une nouvelle vision des rapports conflictuels entre l'Orient et l'Occident au XIX ${ }^{\mathrm{e}}$ siècle. Le projet du canal que le texte évoque dans sa deuxième partie est indissociable des événements qui se déroulent en Europe et dans la région levantine depuis le début du siècle (et du récit). L'œuvre de Traboulsi s'articule de façon à faire de la première partie du texte, essentiellement centrée sur les troubles du Mont-Liban, le début d'un projet de domination monumental qui s'achève par «la percée », puis la «Confluence des deux mers », en Égypte. Les troubles au MontLiban fournissent la pièce manquante dans l'explication de la mise en œuvre du projet malgré les tentatives anglaises de sabotage. Ce n'est qu'à la fin de l'œuvre, avec les deux derniers chapitres, que les pièces du puzzle se rejoignent et que le lecteur mimant l'historien crée des associations entre saint-simonisme, soie (à Lyon et au Mont-Liban), communisme naissant, colonie française en Algérie, Abdelkader, «printemps des peuples » et projet du canal à Suez. Dans la stratégie impérialiste de la France, le canal est, selon Traboulsi, ce changement de cap qui lui permet de réaliser une expansion sans la guerre. La diplomatie et les discours prétendument civilisateurs, œuvres de soie captieuses, arachnéennes et tenaces, auront gain de cause et iront plus loin que les actions répressives, toutes de fer. 


\section{Notes:}

1 Fawwaz Traboulsi, Soie et fer, du Mont-Liban au Canal de Suez, trad. fr. par Marianne Babut et Nathalie Bontemps, Arles, Actes Sud, 2017, Harîr wa hadîd, min jabal Lubnân ilâ qanât al-Suways, pour le titre original, paru en 2012.

2 F. Traboulsi, Soie et Fer, op. cit. Notes des traductrices, p. 9.

3 Née dans les années quatre-vingt-dix, l'histoire connectée est une approche forgée par l'historien indien Sanjay Subrahmanyam qui remet en question la notion de frontières étanches entre les peuples - ou les «aires géographiques »- dans les études historiques. La nouvelle histoire s'écrit essentiellement à travers les allers-retours constants entre les peuples mis en relation. Je remercie Sarga Moussa de m'avoir suggéré ce rapprochement.

4 Titre du dernier chapitre, qui expose au pas de charge des événements qui s'étalent sur un siècle et demi, de 1859 jusqu'en 2004. La nationalisation de la Compagnie du Canal en 1956 y est évoquée aussi bien que l'agression tripartite qu'elle provoque en riposte.

5 En arabe, l'association entre la soie (harîr) et le fer (hadîd) exprime surtout une relation d'antithèse suggérée par la douceur de l'une et la dureté de l'autre. La ressemblance phonétique favorise cet effet d'antinomie.

6 F. Traboulsi, Soie et fer, op. cit., p. 87.

7 F. Traboulsi, Soie et fer, op. cit., p. 195-196.

8 Ibid., p.130.

9 Ibid., p.125-162.

10 Alexandre Gefen, «Le récit biographique à la croisée de l'histoire et de la fiction », dans Histoire et fiction dans les littératures modernes, sous la direction de Richard Jacquemond, Paris, l'Harmattan, 2005, p.61.

11 Rappelons que Traboulsi dédie son œuvre « À la mémoire d'Edward Said ».

12 F. Traboulsi, Soie et fer, op. cit., p. 72.

13 Ibid., p. 49. 
14 Ibid., p. 67.

15 Ibid., p. 120.

16 F. Traboulsi, Soie et fer, op. cit., p. 72.

17 Ibid., p. 75.

18 Ibid., p. 76-77.

19 F. Traboulsi, Soie et fer, op. cit., p. 83.

20 Ibidem.

21 Ibid., p. 92.

22 Ibid., p. 123.

23 Ibid., p. 119.

24 F. Traboulsi, Soie et fer, op. cit., p. 120.

25 Ibid., p. 122. En fait, le terme «d'études» affiché par ladite Société est sujet à caution, cette dernière étant plutôt un instrument d'influence «diplomatique, financier et ingénieurial» improvisé par Enfantin pour agir sur l'Égypte. Voir à ce propos Philippe Régnier, «Le colonialisme est-il un orientalisme? À propos d'Enfantin et de son essai sur la Colonisation de l'Algérie (1843) », dans L'Orientalisme des saint-simoniens, sous la direction de Michel Levallois et Sarga Moussa, Paris, Maisonneuve et Larose, 2006, p. 131-155.

26 Le projet, conçu par la France et finalement abandonné, d'une colonie levantine installée sur les bords du Canal et dirigée par l'émir Abdelkader, est l'exemple d'une information historique marginalisée par l'historiographie occidentale et apportée par Traboulsi comme une pièce manquant au tableau et qui fait sens dans son roman.

27 F. Traboulsi, Soie et fer, op. cit., p. 209. 